\title{
III. MÉTHODES D'ESTIMATION DE LA VALEUR NUTRITIONNELLE DES PROTÉINES
}

\author{
I NTRODUCTION
}

\author{
S. Z. ZELTER \\ Laboratoire de Recherches sur la Conservation et l'Efficacité des Aliments, \\ I6, rue Claude-Bernard. Paris Ve.
}

Il est connu que la technologie appliquée à la fabrication industrielle d'aliments protéiques exerce une action prépondérante sur leur valeur nutritionnelle. L'estimation de celle-ci présente certes des aspects fondamentaux intéressants, mais elle revêt par ailleurs une importance pratique qui préoccupe depuis longtemps les nutritionnistes de divers pays.

Il est en effet indispensable que l'utilisateur de ces produits (fabricant d'aliments composés pour animaux, éleveur) puisse être renseigné rapidement et valablement sur leur qualité, dont l'éventail commercial est très large. Il existe, de ce fait, un besoin impérieux en tests d'appréciation relativement simples et aisément applicables dans des laboratoires de contrôle.

L'épreuve sur l'animal est indubitablement le seul critère décisif, mais elle est très laborieuse et nécessite une installation expérimentale appropriée, fort onéreuse. Aussi, les chercheurs s'emploient-ils depuis de nombreuses années à leur substituer des techniques in-vitro dont les résultats devraient nécessairement, pour être valables, s'harmoniser avec ceux du réactif animal. Dans l'état actuel des choses, la mise au point d'un test de laboratoire polyvalent parait problématique. On en est réduit à rechercher des techniques spécifiques par groupe d'aliments protéiques.

Les recherches exposées plus loin concernent les farines de poisson et le tourteau de soja. Elles ont été effectuées par des épreuves in-vitro selon des méthodes soit biochimiques soit microbiologiques et comparées in-vivo à des mesures sur rat, poussin ou porc, dans deux laboratoires: Le Centre de Recherches sur la Nutrition du C.N.R.S. et le Laboratoire de Recherches sur la Conservation et l'Efficacité des Aliments de I'I.N.R.A. 
Les résultats montrent que si la méthode microbiologique expérimentée procure des valeurs en très bon accord avec celles enregistrées in-vivo sur le rat et le poussin pour les farines de poisson, elle ne fournit pas de relation satisfaisante pour le tourteau de soja. Ce dernier nécessite des tests biochimiques spécifiques distincts de ceux appliqués parfois à des farines animales, comme le prouvent les observations faites sur des porcs.

Il semble donc que dans ce domaine, la continuation persévérante des recherches reste éminemment souhaitable. 